\title{
Multilineage Differentiation of Dental Pulp Stem Cells from Green Fluorescent Protein Transgenic Mice
}

\author{
Brian E. Grottkau, P. Prasad Purudappa, Yun-feng Lin* \\ Department of Orthopaedic Surgery, Mass General Hospital for Children and the Pediatric Orthopaedic Laboratory for Tissue \\ Engineering, Harvard Medical School, Boston, Massachusetts, USA
}

\begin{abstract}
Aim The aim of this study was to confirm the multilineage differentiation ability of dental pulp stem cells (DPSCs) from green fluorescent protein (GFP) transgenic mice. The expression of GFP in DPSCs was also observed during differentiation.
\end{abstract}

Methodology DPSCs were harvested from the dental pulp tissue of transgenic nude mice, and then transferred to osteogenic, adipogenic, and chondrogenic media. The morphological characterization of induced cells was observed by microscopy and histological staining. The expression of marker genes was measured by RT-PCR.

Results The endogenous GFP and multilineage potential of transgenic DPSCs had no influence on each other. Moreover, the results of fluorescence microscopic imaging suggest that there was no significant decline of GFP expression during DPSCs differentiation.

Conclusion As the population of GFP labeled DPSCs can be easily identified, this will be a promising method for tracking DPSCs in vivo.

Keywords dental pulp stem cells, multilineage differentiation, green fluorescent protein

Received Jan. 12, 2010; Revision accepted Feb. 9, 2010

\section{Introduction}

An increasing number of studies has indicated dental pulp is a highly vascularized tissue and contains several niches of stem cells (Sloan et al., 2009; Sumita et al., 2009). The dental pulp stem cells (DPSCs) have multi-potentiality, being capable of differentiating into odontoblasts, osteoblasts, adipocytes, chondrocytes, or neural cells (Iohara et al., 2006; Arthur et al., 2009).

The origin and differentiation of DPSCs is still a matter of some debate (Sloan and Waddington, 2009). Early studies assumed that DPSCs were derived from fibroblast-like cells (Fitzgerald et al., 1990). Gronthos et al. first reported the identification of DPSCs (Gronthos et al., 2000), and subsequent studies reported that the cellular characteristics of DPSCs were similar to bone marrow stem cells (Shi et al., 2001): both DPSCs and bone marrow stem cell express CD44, CD106, CD146, 3G5, and Stro-1 (Ruch, 1998; Gronthos et al., 2002; Sloan and Waddington, 2009). Recent studies suggest that DPSCs might reside in perivascular regions (Shi et al., 2003).

Various attempts have subsequently been made to isolate and characterize progenitor/stem cell populations from adult dental pulp, with the intent of achieving a better defined clonal population of cells (Shi et al., 2005; Wu et al., 2009a). A mesenchymal stem progenitor population, expressing the cell surface receptor Stro-1, has been isolated from adult dental pulp (Braut et al., 2003; Mina et al., 2004; Yang et al., 2007). But the label and tracking of DPSCs in vivo are still a problem to be solved. Green fluorescent protein (GFP) transgenic mice are very useful tools for cell transplantation experiments, since GFP labeled cells can be observed easily and directly without complex procedures or disturbance to cell cultures (Mina and Braut 2004; Balic et al., 2005).

If one is able to determine whether the differentiation of DPSCs has an influence on the 
expression of the endogenous GFP, and if these cells maintained the intensity of GFP expression, one could then apply the GFP-labeled DPSCs into cell transplantation or cell therapy. Taking advantage of improvements in imaging techniques, one could observe the green cells in living organisms and easily confirm the origin of the cells at high resolution. Only the transgenic DPSCs possess the ability to express GFP at a high level during differentiation. Combined with the potential of stem cells, the GFP endogenously labeled DPSCs could be applied broadly in basic research of cell differentiation and tracking.

The purpose of the current study was thus to confirm the multilineage differentiation ability of DPSCs from green fluorescent protein (GFP) transgenic mice, and to observe the expression of GFP in DPSCs during differentiation.

\section{Materials and Methods}

\section{Experimental mouse models}

Eight-week-old GFP transgenic mice were used for this study and their use was in line with the International Guiding Principles for Animal Research. The transgenic mouse lines were produced with an enhanced-GFP (EGFP) cDNA under the control of a chicken $\beta$-actin promoter and cytomegalovirus enhancer. The GFP transgenic mice were provided by the Department of Hematology, State Key Laboratory of Biotherapy of Sichuan University.

Isolation and morphology of DPSCs from GFP

\section{transgenic mouse}

Maxillary and mandibular incisor teeth were dissected from GFP transgenic mice. Then the teeth were washed in sterile phosphate buffered saline (PBS). Dental pulp tissues were carefully harvested and adhered to culture dishes. Five minutes later, the control medium ( $\alpha$-MEM, 10\% fetal bovine serum) was carefully added to submerge the harvested tissue (Lin et al., 2008). These small pieces of tissue were cultured in a humidified atmosphere of $5 \% \mathrm{CO}_{2}$ at $37^{\circ} \mathrm{C}$. When the cells were confluent, they were digested and passaged prior to multilineage differentiation.

\section{Multilineage differentiation of DPSCs}

After culture and expansion in the control medium, the cells were transferred into specific culture media (described in to Table 1) to induce multilineage differentiation (Lin et al., 2006). We performed that least three independent sets of experiments, and each experiment run at least three times.

\section{Cytochemical staining}

The mineralization nodules of osteogenic lineages were stained with alizarin red S (AR-S). The cells were rinsed with PBS and incubated with $40 \mathrm{mmol} \cdot \mathrm{L}^{-1}$ AR-S (pH 4.2) with 10-minute rotation. Then they were rinsed with PBS and observed through a microscope.

After the dental pulp stem cells were in a chondrogenic medium for seven days, the cells were digested, pelleted by centrifugalization at

Table 1 Lineage-specific differentiation induced by medium supplementation

\begin{tabular}{|c|c|c|c|}
\hline Lineage & Medium & Serum & Supplementation \\
\hline Osteogenesis & a-MEM & FBS $(10 \%)$ & $\begin{array}{l}50 \mu \mathrm{mol} \cdot \mathrm{L}^{-1} \text { ascorbate-2-phosphate, } 10 \mathrm{mmol} \cdot \mathrm{L}^{-1} \beta \text {-glycerophosphate, } \\
0.01 \mu \mathrm{mol} \cdot \mathrm{L}^{-1} 1,25 \text {-dihydroxyvitamin } \mathrm{D}_{3}, 1 \% \text { antibiotic/antimycotic }\end{array}$ \\
\hline Chondrogenesis & a-MEM & FBS $(10 \%)$ & $\begin{array}{l}10 \mathrm{ng} \cdot \mathrm{mL}^{-1} \text { TGF- } \beta 1,100 \mathrm{nmol} \cdot \mathrm{L}^{-1} \text { dexamethasone, } 6.25 \mathrm{\mu g} \cdot \mathrm{mL}^{-1} \text { insulin, } \\
50 \mathrm{nmol} \cdot \mathrm{L}^{-1} \text { ascorbate-2-phosphate, } 110 \mathrm{mg} \cdot \mathrm{L}^{-1} \text { sodium pyruvate, } 1 \% \\
\text { antibiotic/antimycotic }\end{array}$ \\
\hline Adipogenesis & a-MEM & FBS $(10 \%)$ & $\begin{array}{l}1 \mu \mathrm{mol} \cdot \mathrm{L}^{-1} \text { dexamethasone, } 10 \mu \mathrm{mol} \cdot \mathrm{L}^{-1} \text { insulin, } 200 \mu \mathrm{mol} \cdot \mathrm{L}^{-1} \text { indo- } \\
\text { methacin, } 0.5 \mathrm{mmol} \cdot \mathrm{L}^{-1} \text { isobutyl-methylxanthine (IBMX), } 1 \% \text { antibiotic } \\
\text { /antimycotic }\end{array}$ \\
\hline Control & a-MEM & FBS $(10 \%)$ & $1 \%$ antibiotic/antimycotic \\
\hline
\end{tabular}


$1200 \mathrm{~g}$ for five minutes, and then cultured as a small mass for another two weeks. The cell sections were stained in toluidine blue for 10 seconds, then washed with water and differentiated in $0.2 \%$ uranyl nitrate until the background appeared colorless.

The adipogenesis was assessed by Oil Red O staining. The cells were rinsed with PBS and fixed in $4 \%$ paraformaldehyde for 15 minutes, stained with $1 \%$ Oil Red $\mathrm{O}$ for 10 minutes. The cells were observed under the fluorescent microscope with red drops (Wu et al., 2009b).

\section{RNA isolation and reverse transcription- polymerase chain reaction}

Total RNA was extracted from all the specimens using the Qiagen RNeasy Mini Kit (Qiagen, US) according to the protocol therein. About $1 \mu \mathrm{g}$ of total RNA was reversed transcribed by murine leukemia virus reverse transcriptase (TaKaRa, Japan), and polymerase chain reaction (PCR) amplification of target message RNA was performed by TaKaRa PCR kit (TaKaRa, Japan). The PCR oligonucleotide primers and annealing temperature are listed in Table 2. The products were electrophoresed on $2 \%$ agarose gels, stained with Ethidium Bromide and visualized with Quantity One software (BIO-RAD).

\section{Results}

\section{Morphology of different passages of DPSCs from GFP transgenic mouse}

After 48-hour culture, the fibroblast-like cells migrated from the attached dental pulp tissue, and all of the attached cells were GFP positive (Figures 1A, B, C). When the cells were confluent, the DPSCs were passaged at the ratio 1:3, and all of the DPSCs were fibroblast-like and GFP positive (Figures 1D, E, F).

\section{Multilineage differentiation of DPSCs}

\section{Osteogenesis}

The 3rd passages of DPSCs were placed in
Table 2 Specific primers for PCR amplification

\begin{tabular}{cl}
\hline Gene & \multicolumn{1}{c}{ Primers } \\
\hline OSX & F: 5'-CACTCACACCCGGGAGAAGA-3' \\
& R: 5'-GGTGGTCGCTTCGGGTAAA-3' \\
OCN & F: 5'-AACGTCCTGGTCACCCTGTATG-3' \\
& R: 5'-GATCTTCTTCACCCGCAGCTT-3' \\
PPAR-y & F: 5'- GACCACTCGCATTCCTTT-3' \\
& R:5'- CCACAGACTCGGCACTCA-3' \\
LPL & F: 5'-AGGGTGAGGAATCTAATG-3' \\
& R: 5'-CAGGTGTTTCAACCGCTA-3' \\
SOX9 & F: 5'- GTTGATCTGAAGCGAGAGGG 3' \\
& R: 5'-CATTGACGTCGAAGGTCTCA 3' \\
Col- II & F: 5'-AAGACCCAGACTGCCTCAAC 3' \\
& R: 5'-TTGGCCCTAATTTTCCACTG 3' \\
GAPDH & F: 5'-CTCACTGGCATGGCCTTCCG -3' \\
& R: 5'-ACCACCCTGTTGCTGTAGCC-3' \\
\hline
\end{tabular}

an osteogenic medium. After culture for 3 weeks, the DPSCs became osteoblast-like in morphology, with a cuboidal shape and formed mineralized nodules (Figure 2A). At the same time, these cells continued to express GFP (Figure 2B). The merged picture (Figure 2C) showed all of the osteogenic differentiated DPSCs also expressing GFP. The mineralized nodules were assessed by alizarin red $\mathrm{S}$ staining (Figure 2D). The results show that the DPSCs continued to express GFP during osteogenic differentiation. In the control group, the DPSCs were cultured in the control medium, and no mineralized nodule formation occurred. To further identify osteogenesis, RT-PCR of OSX and OCN were also performed (Figure 3). Both OSX and OCN were observed in differentiated DPSCs and not observed in the control group.

\section{Chondrogenesis}

To demonstrate chondrogenesis, the DPSCs were placed into chondrogenic medium for 7 days. The cells changed their appearance from fibroblast-like into flat and multi-angled cells (Figure 4A), and the GFP remained positive throughout the process of chondrogenesis and the GFP remained positive throughout the process of chondrogenesis (Figure 4B). The merged picture shows that all of the chondrogenic-differentiated DPSCs also express GFP (Figure 4C). The cells were then pelleted by centrifugation and cultured as a small mass for an additional 3 weeks. Toluidine blue staining was 


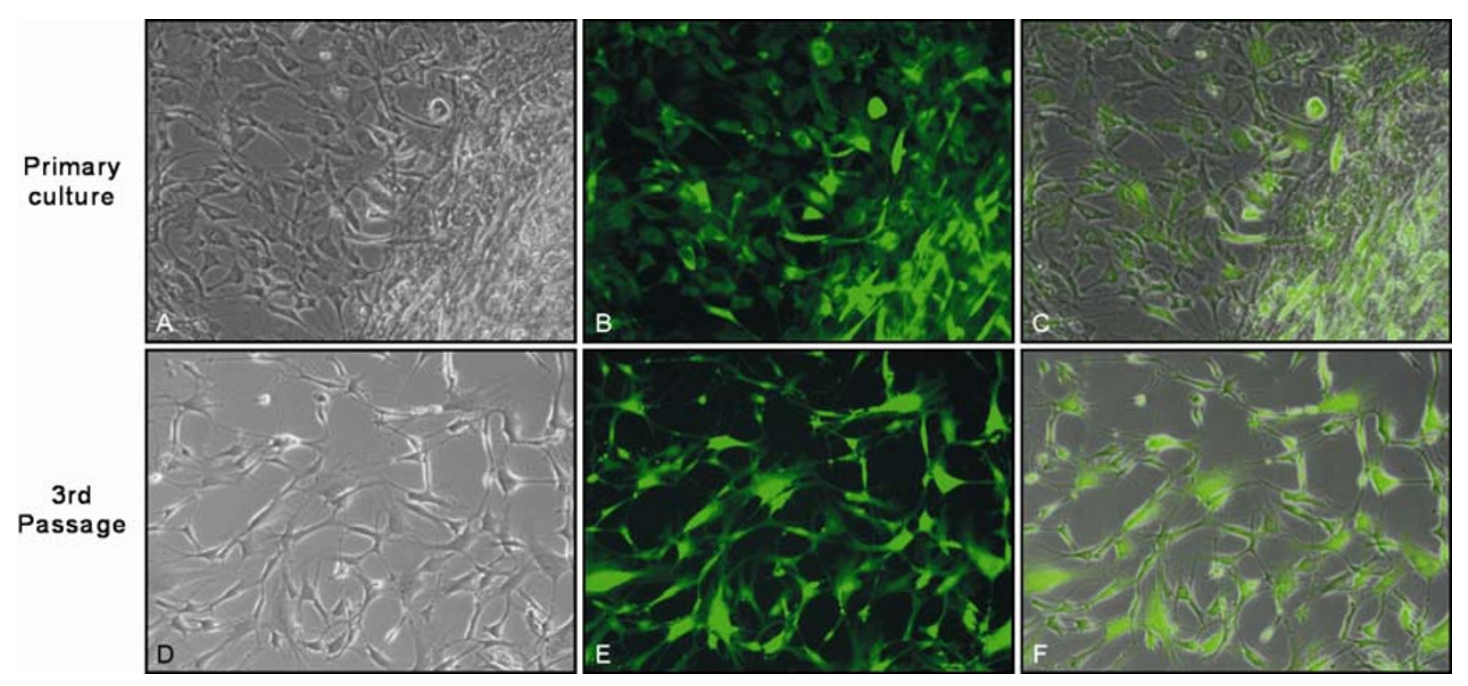

Figure 1 DPSCs were mostly fibroblast-like

In the primary passage of DPSCs, approximately $20 \%$ DPSCs were GFP positive (A, B, C). In the 3rd passage, almost all of the DPSC were GFP positive $(D, E, F)(\times 200)$.

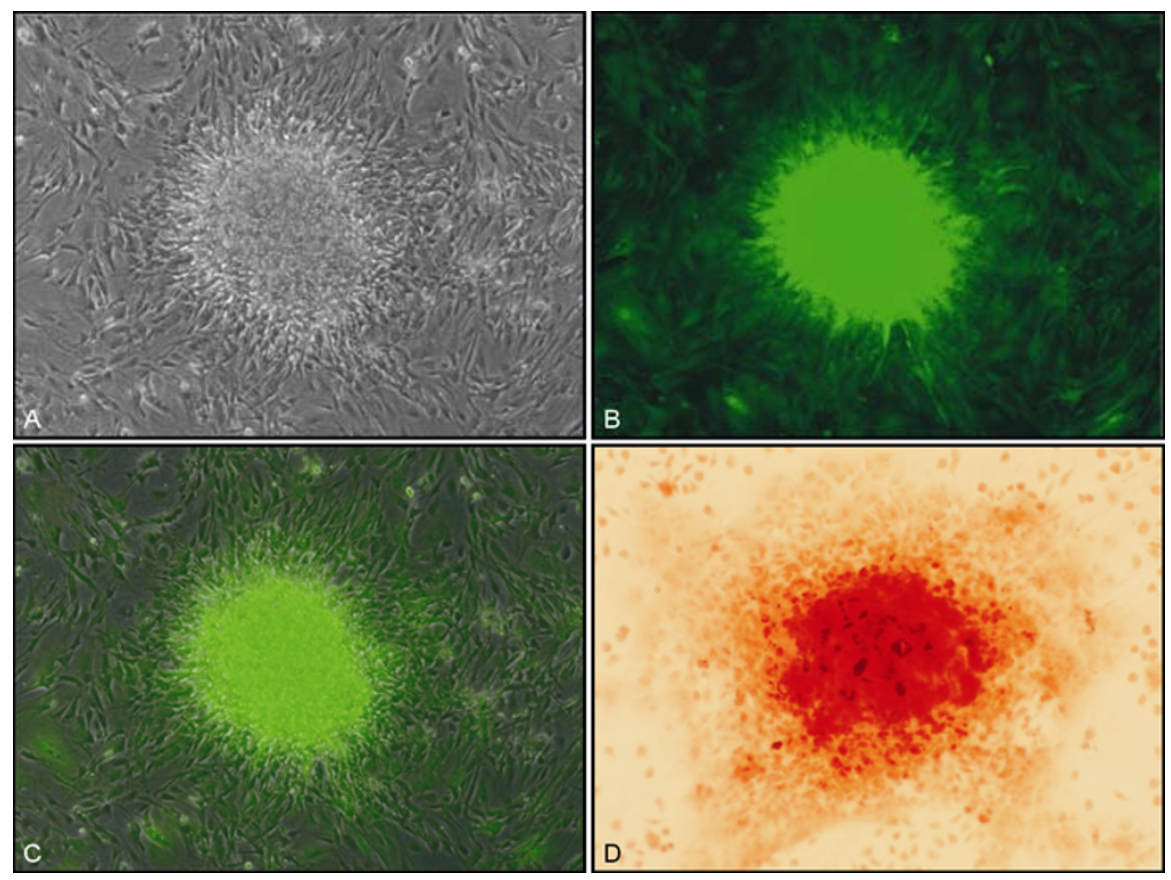

Figure 2 Osteogenic differentiation of DPSCs

(A): After culture in osteogenic medium for 3 weeks, the morphology of DPSCs changed from fibroblast-like to a multilateral and cuboidal form, with a tightly packed arrangement. (B): DPSCs still expressed GFP after osteogenic induction. (C): The merged picture showed all of the osteogenic differentiated DPSCs expressed GFP. (D): Alizarin red S staining of mineralized nodule was positive $(\times 200)$.

then performed and confirmed that the DPSCs differentiated into chondrocyte-like cells (Figure 4D). In the control group, the DPSCs were cultured in the control medium. The toluidine blue staining of these cells was negative.

To further verify chondrogenesis, these cells were also examined by RT-PCR for the expression of specific genes including SOX9 and Col-II
(Figure 5). Both SOX9 and Col- II were observed in differentiated DPSCs, but not in the control group.

\section{Adipogenesis}

Seven days after transfer into adipogenic medium, the morphology of DPSCs changed from an elongated to a rounder morphology and lipid 


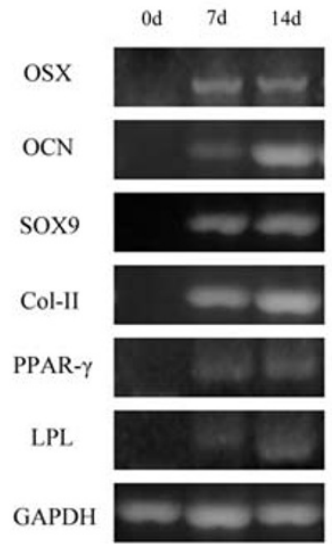

Figure 3 RT-PCR analysis of multilineage differentiation specific genes

The differentiated DPSCs at 7 days and 14 days revealed up-regulated expression of osteogenic, chondrogenic and adipogenic lineage. The control group shows no expression of any of the above genes.

droplets appeared in the cytoplasm (Figure 5A). GFP remained positive in the cytoplasm of DPSCs during the adipogenesis (Figure 5B). Adipogenic differentiation was confirmed by Oil Red $\mathrm{O}$ staining, with lipid droplets demonstrating red fluorescence under the microscope (Figure 5C).
The merged picture suggests that the DPSCs maintain expression of GFP during adipogenesis (Figure 5D). In the control group, the DPSCs were cultured in the control medium, and no lipid drop accumulation was observed.

The expression of adipogenic specific genes PPAR- $\gamma$ and LPL was also examined using RT-PCR (Figure 3). Both PPAR- $\gamma$ and LPL gene expression were observed in differentiated DPSCs and not in the control group.

\section{Discussion}

This study focuses on the tracking and labeling of DPSCs. Under certain conditions, dental pulp produces reparative dentine (Ballini et al., 2007). The DPSCs play an important role in the process, but there is a lack of information about the precise anatomical location of DPSCs in dental pulp (Ballini et al., 2007). This is mainly due to the rarity of stem cells and the absence of specific markers (Shi et al., 2005). The tracking and labeling of dental pulp stem cells is important for in vivo study (Mina and Braut, 2004). Our first goal

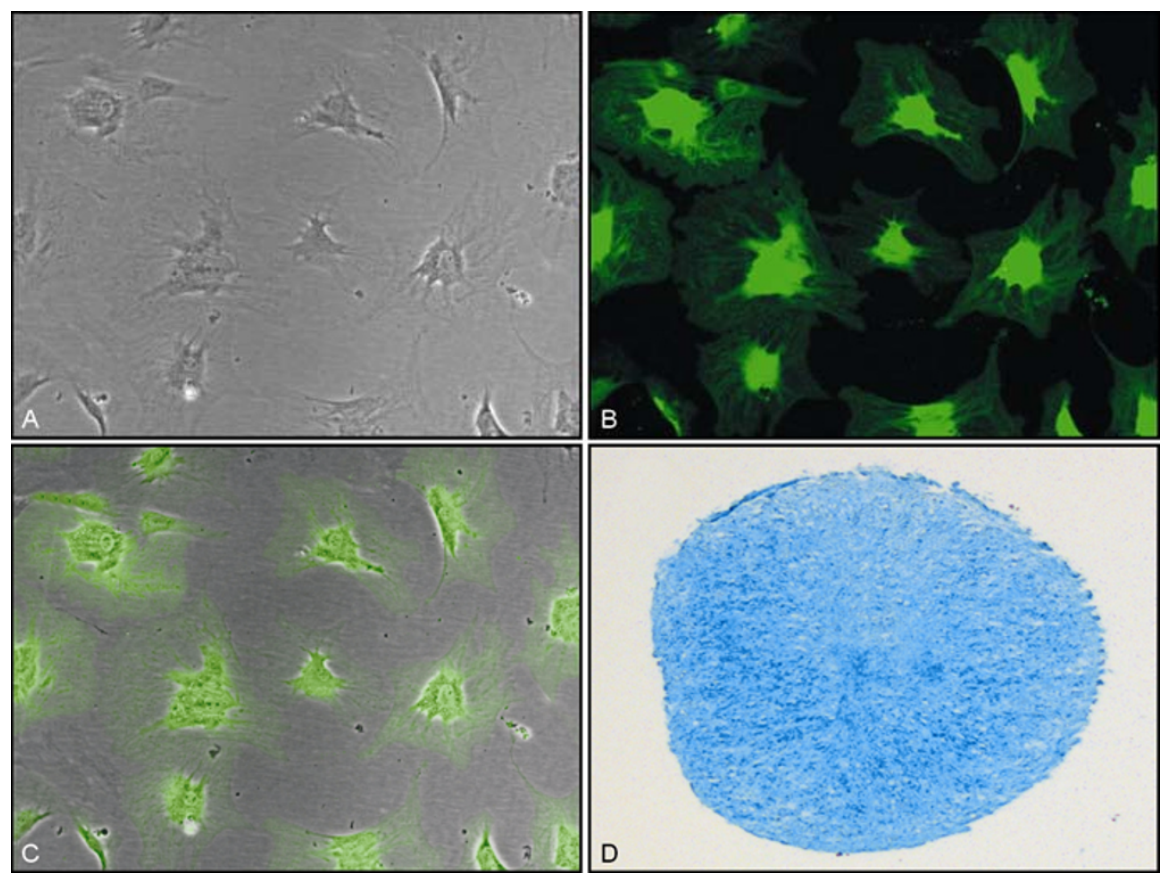

Figure 4 Chondrogenic differentiation of DPSCs

(A): After chondrogenic induction and pellet culture, the cells changed their appearance from fibroblast-like into flat and multi-angle (B): GFP was still positive during chondrogenesis. (C): The merged picture showes that all of the chondrogenic differentiated DPSCs also expressed the GFP $(\times 200)$. (D): The toluidine blue staining of the DPSCs pellet demonstrate that the cells differentiated into chondrocytes Most of the cells were surrounded by proteoglycan after 3 weeks $(\times 40)$. 


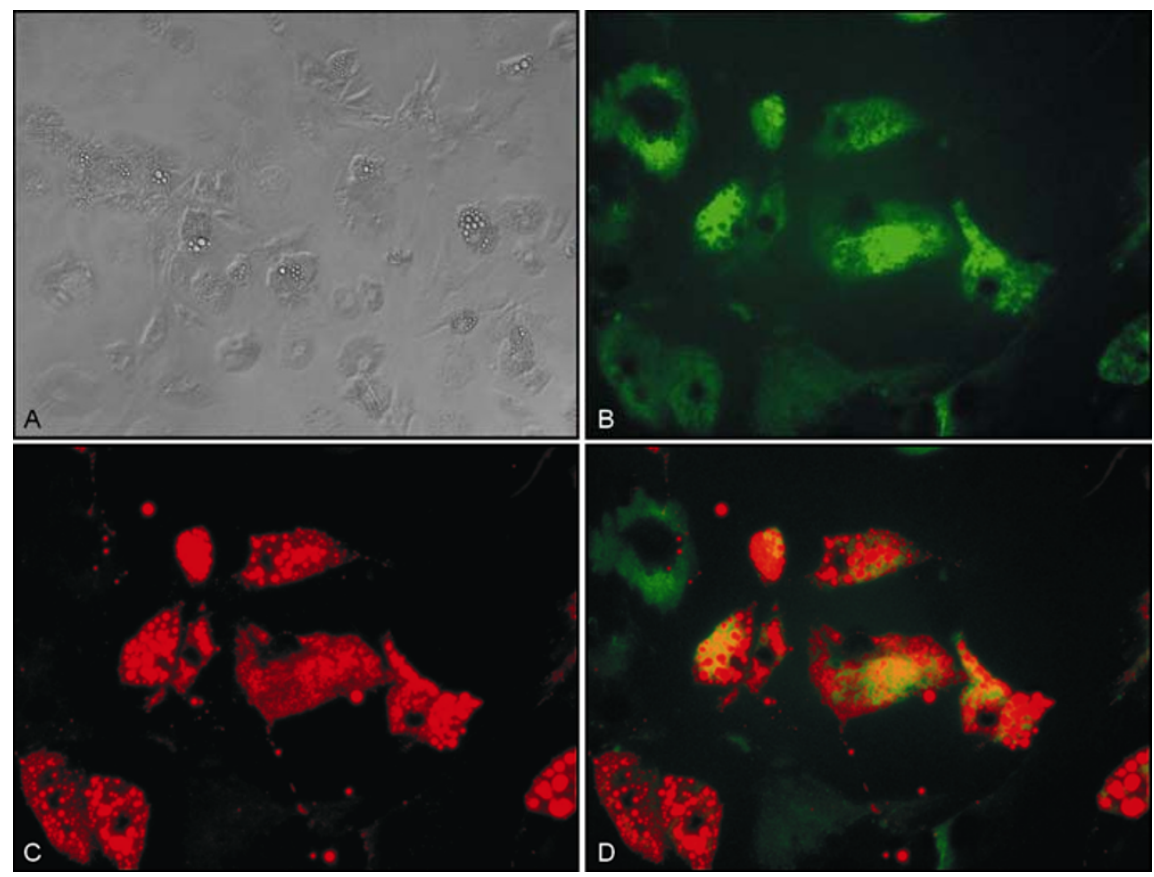

Figure 5 Adipogenic differentiation of DPSCs

(A): DPSCs were incubated in inductive medium for two weeks and lipid accumulation was observed $(\times 200)$. (B): The lipid droplets demonstrated black on green fluorescence in microscopic images. Simultaneously, GFP remained positive during adipogenesis. (C): Oil red O staining results were positive with the lipid dropappearing red on fluorescence microscope. (D): The merged picture proved DPSCs still expressed the GFP during adipogenesis $(\times 400)$.

was to determine whether GFP labeled DPSCs maintained their multiple lineage differentiation ability. Our second goal was to confirm whether the terminal differentiation had a significant influence on GFP expression.

In this study, we demonstrated that DPSCs isolated from GFP transgenic mice maintain the capability of differentiating into adipogenic, osteogenic, and chondrocytic cell types just as normal DPSCs have been reported to do in previous research. DPSCs harvested from dental pulp tissue are fibroblastic in appearance in primary culture and GFP positive. They also had a high proliferation rate in monolayer culture conditions in vitro. GFP expression was maintained until cell senescence or apoptosis. Based on this, dental pulp tissue may represent a promising reservoir of stem cells for tooth tissue engineering and clinical applications.

The GFP transgenic DPSCs may also be an ideal tool for tissue engineering (Mina and Braut 2004). The fluorescent nature of the cells from GFP transgenic mice provides a marker of cell origin, because the cell population can be easily identified and sorted (Hosoya et al., 2007; Balic et al., 2009). In view of this, one can trace and analyze the physiological course of differentiation both in vitro and in vivo. In this study, we obtained valuable information about the GFP transgenic DPSCs differentiation. DPSCs expressing endogenous GFP maintain their ability to differentiate.

With improving microscope technology, one should be able to easily track the GFP-labeled DPSCs in vitro or in vivo. We believe GFP transgenic DPSCs could guarantee the long time maintenance of seeding cells' marker. Future work will focus on culturing GFP transgenic DPSCs committed to odontoblasts within a three-dimensional architecture and implantation of the cell-scaffold system to repair defected dentin.

\section{Acknowledgements}

This work was funded by The Peabody Foundation Inc., the Anthony and Constance Franchi Fund for Pediatric Orthopaedics at the Mass General Hospital for Children, and the National Natural 
Science Foundation of China (30801304), Foundation for the Author of National Excellent Doctoral Dissertation of PR China (FANEDD 200977).

\section{References}

Arthur A, Shi S, Zannettino AC, Fujii N, Gronthos S, Koblar SA (2009). Implanted adult human dental pulp stem cells induce endogenous axon guidance. Stem Cells, 27(9): 2229-2237.

Balic A, Mina M (2005). Analysis of developmental potentials of dental pulp in vitro using GFP transgenes. Orthod Craniofac Res, 8(4): 252-258.

Balic A, Rodgers B, Mina M (2009). Mineralization and expression of Col1a1-3.6GFP transgene in primary dental pulp culture. Cells Tissues Organs, 189(1/2/3/4): 163-168.

Ballini A, De Frenza G, Cantore S, Papa F, Grano M, Mastrangelo F, et al. (2007). In vitro stem cell cultures from human dental pulp and periodontal ligament: new prospects in dentistry. Int J Immunopathol Pharmacol, 20(1): 9-16.

Braut A, Kollar EJ, Mina M (2003). Analysis of the odontogenic and osteogenic potentials of dental pulp in vivo using a Col1a1-2.3-GFP transgene. Int J Dev Biol, 47(4): 281-292.

Fitzgerald M, Chiego DJ Jr, Heys DR (1990). Autoradiographic analysis of odontoblast replacement following pulp exposure in primate teeth. Arch Oral Biol, 35(9): 707-715.

Gronthos S, Brahim J, Li W, Fisher LW, Cherman N, Boyde A, et al. (2002). Stem cell properties of human dental pulp stem cells. J Dent Res, 81(8): 531-535.

Gronthos S, Mankani M, Brahim J, Robey PG, Shi S (2000). Postnatal human dental pulp stem cells (DPSCs) in vitro and in vivo. Proc Natl Acad Sci US A, 97(25): 13625-13630.

Hosoya A, Nakamura H, Ninomiya T, Hoshi K, Yoshiba K, Yoshiba N, et al. (2007). Hard tissue formation in subcutaneously transplanted rat dental pulp. $J$ Dent Res, 86(5): 469-474.

Iohara K, Zheng L, Ito M, Tomokiyo A, Matsushita K, Nakashima M (2006). Side population cells isolated from porcine dental pulp tissue with self-renewal and multipotency for dentinogenesis, chondrogenesis, adipogenesis, and neurogenesis. Stem Cells, 24(11): 2493-2503.

Lin Y, Chen X, Yan Z, Liu L, Tang W, Zheng X, et al. (2006). Multilineage differentiation of adipose-derived stromal cells from GFP transgenic mice. Mol Cell Biochem, 285(1/2): 69-78.

Lin YF, Jing W, Wu L, Li XY, Wu Y, Liu L, et al. (2008). Identification of osteo-adipo progenitor cells in fat tissue. Cell Prolif, 41(5): 803-812.

Mina M, Braut A (2004). New insight into progenitor/stem cells in dental pulp using Col1a1-GFP transgenes. Cells Tissues Organs, 176(1/2/3): 120-133.

Ruch JV (1998). Odontoblast commitment and differentiation. Biochem Cell Biol, 76(6): 923-938.

Shi S, Bartold PM, Miura M, Seo BM, Robey PG, Gronthos S (2005). The efficacy of mesenchymal stem cells to regenerate and repair dental structures. Orthod Craniofac Res, 8(3): 191-199.

Shi S, Gronthos S (2003). Perivascular niche of postnatal mesenchymal stem cells in human bone marrow and dental pulp. J Bone Miner Res, 18(4): 696-704.

Shi S, Robey PG, Gronthos S (2001). Comparison of human dental pulp and bone marrow stromal stem cells by cDNA microarray analysis. Bone, 29(6): 532539.

Sloan AJ, Waddington RJ (2009). Dental pulp stem cells: what, where, how? Int J Paediatr Dent, 19(1): 61-70.

Sumita Y, Tsuchiya S, Asahina I, Kagami H, Honda MJ (2009). The location and characteristics of two populations of dental pulp cells affect tooth development. Eur J Oral Sci, 117(2): 113-121.

Wu G, Deng ZH, Fan XJ, Ma ZF, Sun YJ, Ma DD, et al. (2009a). Odontogenic potential of mesenchymal cells from hair follicle dermal papilla. Stem Cells Dev, 18(4): 583-589.

Wu L, Cai X, Dong H, Jing W, Huang Y, Yang X, et al. (2009b). Serum regulates adipogenesis of mesenchymal stem cells via MEK/ERK dependent PPARgamma expression and phosphorylation. J Cell Mol Med, 2009 Feb 20. [Epub ahead of print].

Yang X, Zhang W, van den Dolder J, Walboomers XF, Bian Z, Fan M, et al. (2007). Multilineage potential of STRO- $1^{+}$rat dental pulp cells in vitro. J Tissue Eng Regen Med, 1(2): 128-135.

*Corresponding author: Yun-feng Lin

Address: Department of Orthopaedic Surgery, Mass General Hospital for Children and the Pediatric Orthopaedic Laboratory for Tissue Engineering, Harvard Medical School, Boston, Massachusetts, USA

Tel: $0016172301799 \quad$ E-mail: ylin15@partners.org 